\title{
COMPARAÇÃO ENTRE A EFICIÊNCIA DE CAPTURA DAS FROTAS DE VARA E ISCA-VIVA E DE CERCO NA PESCARIA DO BONITO LISTRADO (Katsuwonus pelamis): ANÁLISE PRELIMINAR
}

\author{
ALMEIDA, L.R. \& H.A. ANDRADE \\ UNIVALI/CTTMar, Caixa Postal 360, 88302-202-SC \\ E-mail: lau@cttmar.univali.br; humber@cttmar.univali.br
}

\begin{abstract}
RESUMO
A pescaria de bonito listrado (Katsuwonus pelamis) no Brasil é realizada quase que na totalidade por barcos de vara e isca-viva. Uma pequena parcela de embarcações de cerco, que tem como espécie-alvo a sardinha verdadeira (Sardinella brasiliensis), no período de defeso da mesma, tem voltado suas pescarias para o bonito listrado. Seguindo tendências de pescarias realizadas no leste do Atlântico e no Caribe, há atualmente no Brasil um crescente interesse pela substituição de barcos de vara e isca-viva por barcos de cerco, visando aumentar a produção de bonito listrado e atenuar o problema da captura da isca viva. Neste trabalho é feita uma comparação preliminar entre a eficiência de captura desses dois tipos de embarcações, utilizando para isso o poder de pesca e a distribuição de frequência de comprimento dos exemplares capturados. O objetivo foi o de fornecer informações relevantes para que políticas de substituições de frotas não sejam deflagradas sem os conhecimentos necessários. Apesar dos barcos de cerco não estarem adaptados para a pesca de atuns, pôde-se verificar que comparativamente cada um deles tem um poder de pesca até três vezes superior que os de vara e isca-viva. As distribuições de frequência de comprimento obtidas para as pescarias realizadas pelos dois tipos de barcos foram muito semelhantes, demonstrando que o tamanho dos exemplares pode ser, a princípio, independente do artefato de pesca.
\end{abstract}

Palavras-Chave: Katsuwonus pelamis, vara e isca-viva, cerco.

\section{A PRELIMINARY COMPARATIVE ANALYSES ON THE SKIPJACK TUNA (Katswonus pelamis) CATCH EFFICIENCY OF PURSE SEINE AND BAIT BOAT FLEETS}

\begin{abstract}
The fishing of skipjack tuna (Katsuwonus pelamis) in Brazil is almost completely carried out by baitboat fleet. A very small part is done by purse-seine vessels that normally have sardines as a target, but during the annual sardine moratorium, they turn their fishing activities to skipjack tuna. Following the tendencies of the fishing activities realized in the West Atlantic and Caribbean, fisheries in Brazil have shown an interest in the substitution of baitboat by purse-seine vessels, aiming at an increase in the production of skipjack tuna and a reduction of the problem of capturing small fish (bait). In this work a preliminary comparison between the efficiency of the two fishing techniques was carried out, using the fishing power and the length frequency distribution of the specimens captured. The objective was to supply relevant information to that the political substitution of fleets are not being deflagrates without the necessary knowledge. In spite of the purse-seine vessels are not technologically adapted for the fishing of tuna, it can be verified that comparatively each one of them has the fishing power up to three times greater than those baitboat vessels. The
\end{abstract}


length frequency distribution obtained by the fisheries using both types of boat were very similar, demonstrating that there maybe no significant differences in the selectivity of both gears.

Key-Words: Katsuwonus pelamis, baitboat, purse-seine.

\section{INTRODUÇÃO}

A pescaria de bonito listrado (Katsuwonus pelamis), realizada no oeste do Atlântico, começou a se desenvolver na década de 1950 com barcos de vara e isca-viva, e a partir da década de 1960 com barcos de cerco (Delgado de Molina et al. 1999). Dentre essas duas artes de pesca, a contribuição dos barcos de cerco é consideravelmente menor que a de vara e isca-viva, no oeste do Atlântico. Segundo dados da ICCAT, cerca de $86 \%$ do bonito listrado capturado nesta região é proveniente da pesca de vara e isca-viva, e somente $13 \%$ de cerco. Outras artes de pesca contribuíram com 1\% (ICCAT, 2000). Entre os países que atuam nesta região, o Brasil é o mais importante, perfazendo em torno de $85 \%$ do capturado nos últimos anos, quase que na totalidade por vara e isca-viva (ICCAT, 2000). Somente a partir da metade da década de 90 , barcos de cerco brasileiros que atuam sobre a sardinha verdadeira (Sardinella brasiliensis) começaram a capturar eventualmente bonito listrado em épocas de defeso da sardinha (ex: $15 / 12$ a 15/03). No entanto, o desembarque resultante desta arte de pesca é ainda muito pequeno e restrito a um período específico.

Houve e ainda há uma tendência clara de substituição da frota de vara e isca-viva pela frota de cerco nas pescarias de atuns no leste do Atlântico, no Caribe e na costa da Venezuela. O Brasil é o único país do Atlântico Sul em que a captura de bonito é ainda proveniente quase que na totalidade da frota de vara e iscaviva. No entanto, nos últimos anos, aumentou o interesse de vários segmentos pesqueiros em implantar efetivamente uma frota de cerco voltada a captura de atuns no Brasil. Este requerimento ganha forças na idéia de que com uma substituição gradativa de pelo menos parte das embarcações de vara e isca-viva, por embarca- ções de cerco, haveria uma atenuação do problema da captura de isca-viva. A captura da isca-viva em regiões costeiras e em áreas de preservação, por parte das embarcações atuneiras, tem gerado amplas discussões em âmbitos sociais, políticos e científicos (Lin, 1992; Dos Santos \& Rodrigues-Ribeiro, 2000; GEP/CTTMar, 2001). A isca-viva é também tida hoje como um fator limitante da pescaria do bonito, pois torna a atividade bem mais cara uma vez que os pescadores despendem muito tempo e recurso para iscar o barco antes de se dirigirem para a pescaria propriamente dita.

A liberação de licenças para embarcações de redes de cerco, apesar dos interesses atuais, deve ser avaliada previamente, uma vez que tais embarcações têm normalmente um poder de pesca elevado, e acarretariam em um incremento significativo do esforço de pesca atual. Portanto, a importância de um estudo referente à comparação entre essas duas artes de pesca é indiscutível, principalmente para subsidiar liberações e substituições de licenças. Sendo assim, o presente trabalho teve como objetivo apresentar o poder de pesca dos barcos de cerco em relação aos de vara e iscaviva, além de uma análise superficial das possíveis diferenças entre a distribuição de frequência de comprimento dos exemplares capturados por essas artes de pesca.

\section{MATERIAIS E MÉTODOS}

Foram utilizados dados adquiridos em descarregamentos de bonito listrado realizados em 1999 e 2000 no porto de Itajaí-SC.

As comparações foram feitas para barcos que pescaram em uma mesma área e em datas muito próximas, visando minimizar vícios derivados de variações naturais da disponibilidade do recurso. O posicionamento das 
embarcações foi mapeado em uma grade de $1^{\circ}$ lat. $\times 1^{\circ}$ long. Uma vez que foram analisadas unicamente operações de pesca que ocorreram em pontos muito próximos, somente três comparações foram possíveis. Esse pequeno tamanho amostral, não permitiu que uma análise estatística pudesse ser feita, e portanto os resultados obtidos são apenas preliminares e descritivos.

O poder de pesca das embarcações de cerco $(P c)$, em relação às embarcações de vara e isca-viva foi estimado através de:

$P C=U c / U v$

onde Uce Uv são as capturas por unidade de esforço (CPUE) das embarcações de cerco e vara e isca-viva, respectivamente. As CPUEs de uma frota $i$ qualquer $(U i)$ podem ser obtidas por:

$U i=C i / f i$

onde Cie fi são a captura e o esforço da frota $i$. Este procedimento segue a metodologia tradicional exposta em Gulland (1983). Para os cálculos foram utilizadas as duas unidades de esforço tradicionalmente adotadas para essa pescaria, dias de mar e dias de pesca (GEP/ CTTMar, 2001).

Além das CPUEs, foram comparadas também as distribuições de freqüência de comprimento de capturas derivadas dos dois tipos de frotas. Essa análise foi possível em somente um caso, onde os barcos pescaram em uma mesma área e na mesma época, e na qual houve ainda amostragem biológica nos desembarques.

As distribuições de freqüência foram apresentadas em números absolutos, em porcentagem e em número de peixes capturados por dia de pesca.

\section{RESULTADOS E DISCUSSÃO}

Os dados sumarizados são apresentados na tabela 1. Pode-se verificar que os barcos de cerco normalmente apresentam menor número de dias de mar por viagem, e que este valor é muito semelhante ao número de dias de pesca. Já os barcos de vara e isca-viva apresentaram grande variação quanto aos dias de mar e de pesca, provavelmente devido ao fato de que essa arte de pesca depende essencialmente da captura da isca-viva, realizada pelos próprios barcos. Sendo assim, essa captura pode ser rápida, como pode levar muitos dias, retardando a captura do bonito listrado e aumentando o tempo e o custo da viagem. A CPUE da frota de cerco apresentou-se sempre superior à de vara e isca-viva, apesar das duas serem bastante variáveis (Tab. 1).

O poder de pesca dos barcos de cerco, baseado no esforço em dias de mar, variou entre 1,72 e 3,03 , e o baseado em dias de pesca

Tabela 1: Comparações entre pescarias de bonito (Katsuwonus pelamis) realizadas por embarcações de cerco e de vara e isca-viva. As latitudes e longitudes indicam o centro do quadrado de $10 \times 10$ na qual foram realizadas as pescarias. As CPUEs foram calculadas em toneladas por dias de mar (t/dm) e toneladas por dias de pesca (t/dp).

\begin{tabular}{|c|c|c|c|c|c|c|c|c|c|c|c|c|}
\hline Barco & Tipo de pesca & $\begin{array}{l}\text { Lat. } \\
\left({ }^{\circ} \mathrm{S}\right)\end{array}$ & $\begin{array}{l}\text { Long. } \\
\left({ }^{\circ} W\right)\end{array}$ & Prof. & $\begin{array}{l}\text { Data da } \\
\text { pescaria }\end{array}$ & $\begin{array}{c}\text { Esforço } \\
\text { (dias de } \\
\text { mar) }\end{array}$ & $\begin{array}{l}\text { Esforço } \\
\text { (dias de } \\
\text { pesca) }\end{array}$ & $\begin{array}{l}\text { Captura } \\
\text { (t) }\end{array}$ & $\begin{array}{l}\text { CPUE } \\
(\mathrm{t} / \mathrm{dm})\end{array}$ & $\begin{array}{l}\text { CPUE } \\
\text { (t/dp) }\end{array}$ & $\begin{array}{l}\text { Poder de } \\
\text { pesca (com } \\
\text { dias de } \\
\text { mar) }\end{array}$ & $\begin{array}{c}\text { Poder de } \\
\text { pesca } \\
\text { (com dias de } \\
\text { pesca) }\end{array}$ \\
\hline Felipe Jorge & cerco & 29,5 & 48,5 & 130 & $\begin{array}{c}09 \mathrm{a} \\
13 / 01 / 1999\end{array}$ & 4 & 2 & 27 & 6,75 & 13,5 & 1,72 & 2,7 \\
\hline $\begin{array}{c}\text { Águia Dourada } \\
\text { XIII } \\
\end{array}$ & vara e isca-viva & 29,5 & 48,5 & $\begin{array}{l}240- \\
320 \\
\end{array}$ & $\begin{array}{c}04 \mathrm{a} \\
18 / 01 / 1999 \\
\end{array}$ & 14 & 11 & 55 & 3,93 & 5 & & \\
\hline Da Hora XII & cerco & 27,5 & 47,5 & $75-80$ & $\begin{array}{c}27 / 01 \text { a } \\
01 / 02 / 1999\end{array}$ & 4 & 4 & 13 & 3,25 & 3,25 & 2,17 & 2,17 \\
\hline $\begin{array}{c}\text { Schimitt } \\
\text { Hasegawa VII }\end{array}$ & vara e isca-viva & 27,5 & 47,5 & 75 & $\begin{array}{c}29 / 01 \text { a } \\
02 / 02 / 1999\end{array}$ & 4 & 4 & 6 & 1,5 & 1,5 & & \\
\hline Dom Manuel IV & cerco & 27,5 & 48,5 & $55-70$ & $\begin{array}{c}22 \mathrm{a} \\
26 / 01 / 2000\end{array}$ & 3 & 2 & 28 & 9,33 & 14 & 3,03 & 1,05 \\
\hline $\begin{array}{c}\text { Águia Dourada } \\
\text { XIII } \\
\end{array}$ & vara e isca-viva & 27,5 & 47,5 & 80 & $\begin{array}{c}18 \mathrm{a} \\
31 / 01 / 2000 \\
\end{array}$ & 13 & 3 & 40 & 3,08 & 13,3 & & \\
\hline
\end{tabular}


variou entre 1,05 e 2,7 (Tab. 1). Devido ao pequeno número amostral utilizado neste estudo, deve-se analisar com cautela os resultados obtidos, mas tudo indica que os barcos de cerco apresentam um poder de captura pelo menos duas vezes maior que os de vara e iscaviva. Sendo assim, em uma substituição de barcos de vara e isca-viva por de cerco, para cada barco de cerco introduzido na pesca, deveriam sair aproximadamente três de vara e isca-viva. Essa decisão cautelar estaria em conivência com códigos pesqueiros de conduta precautória diante das incertezas das estimativas.

Vale salientar que os barcos de cerco analisados no presente trabalho estão voltados principalmente à captura da sardinha. Segundo IBAMA (1994) e Occhialini (2000) as características físicas médias dessas embarcações, em Santa Catarina, são: 74,92 TBA, potência do motor $326,44 \mathrm{Hp}$ e comprimento $23,8 \mathrm{~m}$. Sendo assim, os barcos dessa frota são relativamente pequenos, com petrechos não adaptados e com pescadores sem experiência na captura de atuns, apresentando um poder de pesca provavelmente menor do que o potencial da embarcação. Portanto, nossas estimativas de poder de pesca são válidas para as embarcações de cerco ora atuantes no Brasil. Da mesma forma, extrapolações de valores para outras categorias de embarcações (e.g. TBA $>80$ ) devem ser também cautelosas. A necessidade de uma política precautória ganha reforço no fato de que uma frota/artefato novo em uma pescaria teria a tendência de aumentar a sua eficiência rapidamente nos primeiros anos, em função do aprendizado dos pescadores (Hilborn e Walters, 1992). Há inclusive evidências disso para a própria pescaria de vara e isca-viva no início de sua implementação no Brasil (Andrade, dados não publicados). Portanto, é esperado que nos primeiros anos de funcionamento, uma nova frota de cerco passe a ter um poder de pesca maior do que o calculado nesse trabalho.

Quanto a análise da distribuição de frequência, ambas foram muito semelhantes
(Fig. 1), demonstrando que o tamanho dos exemplares capturados pode a princípio ser independente do artefato de pesca utilizado. As características do próprio cardume quanto à composição de tamanho podem ser determinantes para a estrutura de comprimento da captura obtida. No entanto, a aquisição de mais informações é necessária para que análises adequadas possam ser feitas. De qualquer forma, é interessante notar que apesar dos dois artefatos atingirem percentualmente as mesmas parcelas da população (fig. 1 a), o impacto por dia é muito maior no caso da pesca de cerco (fig. $1 \mathrm{c}$ ).

\section{REFERÊNCIAS BIBLIOGRÁFICAS}

Dos Santos, R.C. \& M. Rodrigues-Ribeiro. 2000. Demanda de iscas vivas para a frota atuneira catarinense na safra de 1998/1999: CPUE, composição e distribuição das capturas.Notas Téc. FACIMAR, 4:97-101.

Delgado de Molina, A.; Fonteneau, A.; Pallarés, P.; Ariz, J.; Gaertner, D. \& C. Santana. 1999. Atlantic tropical tuna fisheries: general overview. SCRS/98/36. Vol.XLIX (3) 241-252. GEP/CTTMar, 2001. Relatório Final do Convênio MA/UNIVALI. MA/SARC/03/2000, 67p.

Gulland, J. A. 1983. Fish Stock Assessment A Manual of Basic Methods. John Wiley \& Sons. Chichester. 223 pp.

Hilborn, R. \& C.J. Walters. 1992. Quantitative Fish Stock Assessment - Choice, Dynamics and Uncertainty. Chapman \& Hall. New York. 570 pp.

IBAMA. 1994. Coleção Meio Ambiente: Atuns e afins e da sardinha. Série estudos pesca, 11. MMA/IBAMA, Brasília, 200p.

ICCAT. 2000. Detailed Report on Skipjack Tuna. SCRS/99/21. Vol. LI. 132-220.

Lin, C.F. 1992. Atuns e Afins: Estimativa da Quantidade de Isca-Viva Utilizada pela Frota Atuneira. Coleção Meio Ambiente, Série Estudos de Pesca $n^{\circ}$ 6. IBAMA, Brasília, DF, 80p. 

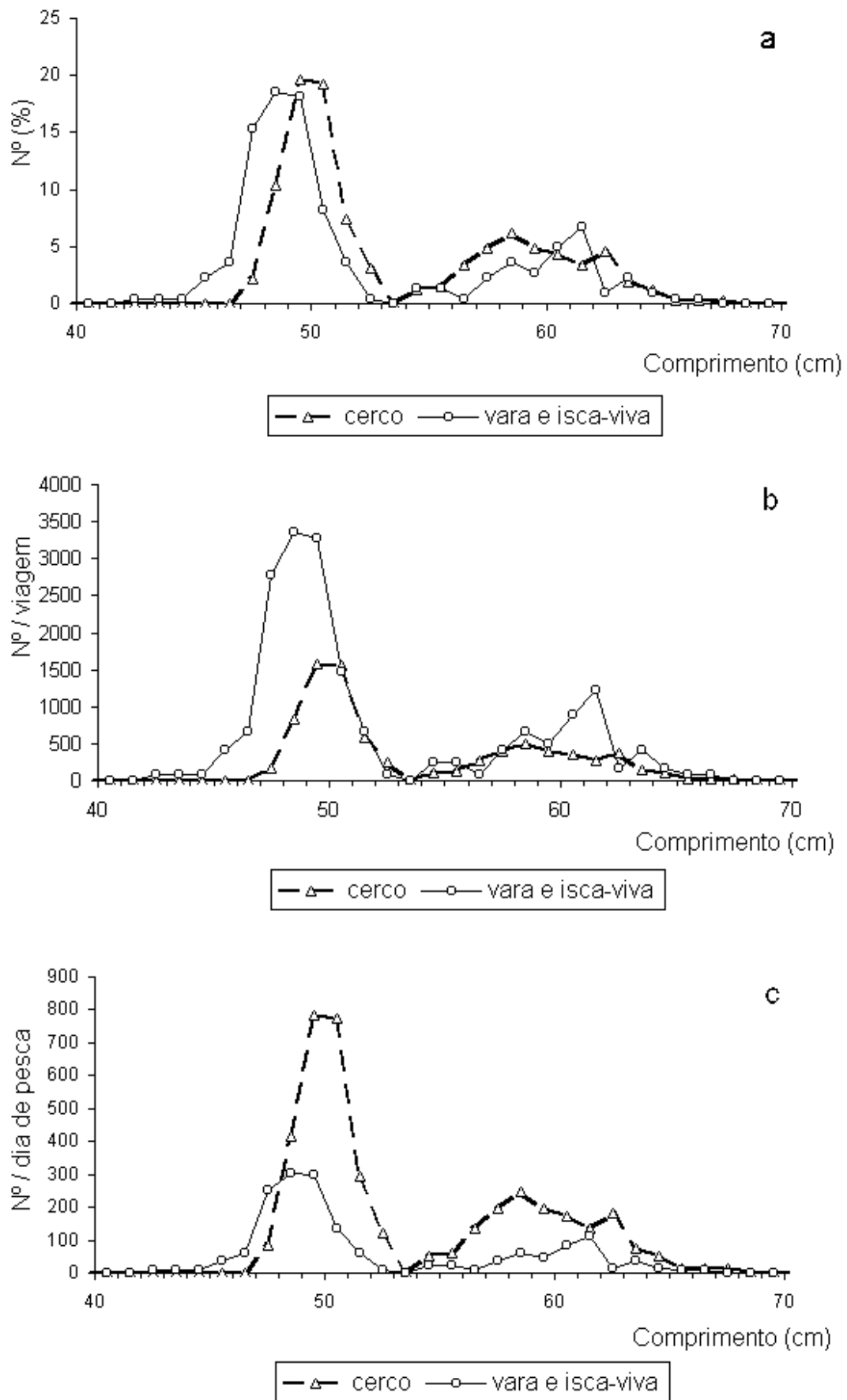

Fig. 1 - Distribuição de frequência de comprimento do bonito listrado (Katsuwonus pelamis) capturado por barcos de cerco e de vara e isca-viva. (a) porcentagem, (b) número capturado por viagem, e (c) número capturado por dia de pesca. 
ALMEIDA, L.R. \& H.A. ANDRADE: Eficiência das frotas na captura de atuns.

Occhialini, D.S. 2000. Descrição e dinâmica da frota de traineiras no porto de Itajaí, SC, entre 1997 e 1999. Monografia, UNIVALI, Itajaí, 65p. 